\title{
Nonautonomous Differential Equations in Banach Space and Nonrectifiable Attractivity in Two-Dimensional Linear Differential Systems
}

\author{
Siniša Miličićc and Mervan Pašićc \\ University of Zagreb, Faculty of Electrical Engineering and Computing, Department of Mathematics, 10000 Zagreb, Croatia \\ Correspondence should be addressed to Siniša Miličić; sinisa.milicic@fer.hr
}

Received 24 January 2013; Accepted 18 March 2013

Academic Editor: Elena Braverman

Copyright (C) 2013 S. Miličić and M. Pašić. This is an open access article distributed under the Creative Commons Attribution License, which permits unrestricted use, distribution, and reproduction in any medium, provided the original work is properly cited.

We study the asymptotic behaviour on a finite interval of a class of linear nonautonomous singular differential equations in Banach space by the nonintegrability of the first derivative of its solutions. According to these results, the nonrectifiable attractivity on a finite interval of the zero solution of the two-dimensional linear integrable differential systems with singular matrix-elements is characterized.

\section{Introduction}

Let $t_{0}>0$ and $\mathbb{X}$ a given real or complex Banach space with the zero $\mathbf{0} \in \mathbb{X}$, and let $L(\mathbb{X})$ denote the space of all linear bounded operators from $\mathbb{X}$ into $\mathbb{X}$. We consider the linear nonautonomous differential equation in $\mathbb{X}$ as

$$
\frac{d \mathbf{x}}{d t}=A(t) \mathbf{x}, \quad t \in\left(0, t_{0}\right], \quad \mathbf{x}\left(t_{0}\right)=\mathbf{x}_{0} \in \mathbb{X}
$$

where $\mathbf{x}=\mathbf{x}(t), \mathbf{x} \in C^{1}\left(\left(0, t_{0}\right] ; \mathbb{X}\right), A=A(t)$ is an operatorvalued function defined on interval $\left(0, t_{0}\right]$ with the value in $L(\mathbb{X})$ and $A \in C\left(\left(0, t_{0}\right] ; L(\mathbb{X})\right)$. If $\mathbb{X}=\mathbb{R}^{N}$, then (1) becomes a linear nonautonomous differential system.

As a basic result, in Theorem 1, we state the existence and uniqueness of a solution of (1) which is proved by using a fixed-point theorem in Fréchet spaces for an $\alpha$-contraction mapping. Next, in Theorems 2 and 3, we give a necessary and sufficient condition for the nonintegrability of $\left\|\mathbf{x}^{\prime}(t)\right\|_{\mathbb{X}}$ on $\left(0, t_{0}\right]$ provided $\|A(t)\|$ blows up near $t=0$ and $\|A(t) x\|$ allows a precise asymptotic behaviour near $t=0$. Based on these results, in the case when $\mathbb{X}=\mathbb{R}^{2}$, we study the so-called nonrectifiable attractivity of the zero solution (see Definition 4) of the so-called linear integrable twodimensional system (see Definition 5) as follows:

$$
\begin{array}{r}
\frac{d \mathbf{x}}{d t}=\left[\begin{array}{cc}
h(t)+\mu g(t) & -v g(t) \\
\rho g(t) & h(t)
\end{array}\right] \mathbf{x}, \\
t \in\left(0, t_{0}\right], \mathbf{x}\left(t_{0}\right)=\mathbf{x}_{0} \in \mathbb{X},
\end{array}
$$

with $\mu, \rho$, and $\nu$ some real constants.

Precisely, as a kind of singular behaviour near $t=0$ of all solutions $x(t)$ of linear integrable system (2), in Theorem 6, we involve on the matrix elements $h(t)$ and $g(t)$ a necessary and sufficient condition for the infiniteness of the length of every solution curve of $x(t)$ (in our best knowledge, it is the first paper dealing with this kind of problems). Theorem 6 is a consequence of the precise asymptotic formula for all solutions $x(t)$ near $t=0$ of integrable differential system (2) presented in Lemmas 11 and 12. Of course, instead of interval $\left(0, t_{0}\right]$ and $t \rightarrow 0$, we can also state our main results on $\left[t_{0}, \infty\right)$ and $t \rightarrow \infty$.

In applications, the last years have seen an increasing interest in the analysis of differential equations and systems on finite time intervals. This is because the Finite-Time Stability (FTS) and the Finite-Time Lyapunov Exponent (FTLE) were introduced, respectively, in the control of systems within a finite time as well as in the Lagrangian Coherent Structure 
(LCS) on finite-time intervals in fluid, ocean, and atmosphere dynamics [1-3] and in the biological application [4]. It includes the time-varying vector fields known only on a finite-time interval, but not on the whole half line $t \geq t_{0}$.

In the theory of differential equations, the importance of studying the different kind of asymptotic behaviours of the nonautonomous linear differential systems comes from their application in the study of asymptotic and oscillatory behaviour of the second and higher order ordinary differential equations. For instance, in [5], authors study the asymptotic behaviour near $t=\infty$ of oscillatory solutions of the nonlinear second-order differential equation $\left(p(t) u^{\prime}\right)^{\prime}=$ $p(t) f(u)$ via the asymptotic formula for solutions of an auxiliary linear differential system having elements which are absolutely continuous functions on $\left[t_{0}, \infty\right)$. In [6], authors derive a precise asymptotic behaviour near $t=\infty$ of solutions $u(t)$ of the third-order nonlinear differential equation $u^{\prime \prime \prime}+$ $((m+1) / 2) u u^{\prime \prime}+m\left(1-u^{\prime 2}\right)+M\left(1-u^{\prime}\right)=0$ such that $u(0)=a, u^{\prime}(0)=b, u^{\prime}(\infty)=1$, and $0<u^{\prime}<1$ on $[0, \infty)$, by using the asymptotic solution formula for the corresponding linear differential system based on Hartman and Wintner's asymptotic integration (see $[7,8]$ and for its generalization $[9,10])$. Similarly, in [11], authors prove an asymptotic solution formula for the second-order nonlinear differential equations $\left(p u^{\prime}\right)^{\prime}+q u=g\left(t, u, u^{\prime}\right)$ depending on the asymptotic behaviour of fundamental solutions of the corresponding homogeneous equation $\left(p z^{\prime}\right)^{\prime}+q z=$ 0 . On certain types of asymptotic behaviour of linear and nonlinear differential and integro-differential systems, we refer reader to some recently published papers [12-14] and references therein. The attractivity of solutions of scalar delay differential equations is widely studied and it is important in mathematical biology; see, for instance, [15] and references therein.

This paper is mainly based on a part of the P.h.D degree thesis [16] of the first author.

\section{Statement of the Main Results}

Preliminarily, we state the following auxiliary result.

Theorem 1. For each $\mathbf{x}_{0} \in \mathbb{X}$, there exists a unique solution $\mathbf{x}$ of (1).

Next, we derive a necessary condition for a kind of singularities of (1) at $t=0$ in terms of singular behaviour of the operator norm of $A(t)$ near $t=0$.

Theorem 2. Let there exist a solution $\mathbf{x}$ of (1) such that $\|\mathbf{x}(t)\|_{\mathbb{X}} \rightarrow 0$ as $t \rightarrow 0$. If $\|d \mathbf{x} / d t\|_{\mathbb{X}} \notin L^{1}\left(0, t_{0}\right)$, then one has:

$$
\lim \sup _{t \rightarrow 0}\|A(t)\|=\infty .
$$

Thus, if (3) is not satisfied, then the integrability of $\|d \mathbf{x} / d t\|_{\mathbb{X}}$ occurs for all solutions $\mathbf{x}$ of (1), which is out of our interest. All results from this section will be proved in the next sections.
At the second, we impose on the operator-valued function $A(t)$ the following structural hypotheses:

$\left(H_{1}\right)$ there are positive constants $c_{0}, c_{1}$ and a positive real function $\omega=\omega(t)$ such that

$$
\begin{array}{r}
c_{0} \omega(t)\|x\|_{\mathbb{X}} \leq\|A(t) x\|_{\mathbb{X}} \leq c_{1} \omega(t)\|x\|_{\mathbb{X}} \\
\forall t \in\left(0, t_{0}\right], \forall x \in \mathbb{X} ;
\end{array}
$$

$\left(H_{2}\right)$ the solution of (1) is given by

$$
\mathbf{x}(t)=U(t) \mathbf{x}_{0}, \quad t \in\left(0, t_{0}\right]
$$

where $U=U(t), U:\left(0, t_{0}\right] \rightarrow L(\mathbb{X})$, is an operator-valued function called the fundamental solution, such that

$$
\|U(t)\| \longrightarrow 0 \quad \text { as } t \longrightarrow 0,
$$

and there are positive constants $c_{2}, c_{3}$ and a positive real function $a=a(t)$ such that

$$
\begin{array}{r}
c_{2} a(t)\|x\|_{\mathbb{X}} \leq\|U(t) x\|_{\mathbb{X}} \leq c_{3} a(t)\|x\|_{\mathbb{X}} \\
\forall t \in\left(0, t_{0}\right], \forall x \in \mathbb{X} .
\end{array}
$$

Such hypotheses on (1) imply the following properties of every solution $\mathbf{x}$ and its $d \mathbf{x} / d t$.

Theorem 3. Let the hypotheses $\left(H_{1}\right)-\left(H_{2}\right)$ be fulfilled. Then, for every $\mathbf{x}_{0} \in \mathbb{X}$, the corresponding solution $\mathbf{x}$ of (1) satisfies

(i) $\|\mathbf{x}(t)\|_{\mathbb{X}} \rightarrow 0$ as $t \rightarrow 0$;

(ii) $\mathbf{x}$ is an injective function on $\left(0, t_{0}\right]$ if and only if $\mathbf{x}_{0}$ is not an eigenvector of $U^{-1}(s) U(t)$ with an eigenvalue 1 for all $s, t \in\left(0, t_{0}\right], s \neq t$;

(iii) $\|d \mathbf{x} / d t\|_{\mathbb{X}} \notin L^{1}\left(0, t_{0}\right)$ if and only if $\omega a \notin L^{1}\left(0, t_{0}\right)$.

The meaning and importance of hypotheses $\left(H_{1}\right)-\left(H_{2}\right)$ as well as the conclusions (i)-(iii) of Theorem 3 will be verified by Theorem 6 , where $\mathbb{X}=\mathbb{R}^{2}$ and (1) is a large class of twodimensional linear differential systems.

The second aim of the paper is to use previous theorem in the study of the rectifiable and nonrectifiable attractivity of zero solution of two-dimensional linear differential system as

$$
\begin{gathered}
\mathbf{x}^{\prime}=A(t) \mathbf{x}, \quad t \in\left(0, t_{0}\right], \\
\mathbf{x}\left(t_{0}\right)=\mathbf{x}_{0} \in \mathbb{R}^{2}, \\
\mathbf{x} \in C^{1}\left(\left(0, t_{0}\right] ; \mathbb{R}^{2}\right),
\end{gathered}
$$

where $t_{0}>0$ and the prime denotes $d / d t, \mathbf{x}=\mathbf{x}(t)=$ $(x(t), y(t))$ and the matrix-valued function $A=A(t), A$ : $\left(0, t_{0}\right] \rightarrow \mathscr{M}_{2}(\mathbb{R})$ (where $\mathscr{M}_{2}(\mathbb{R})$ denotes the space of real $2 \times 2$ matrices), is continuous on $\left(0, t_{0}\right]$. Therefore, we may apply previous theorems to system (8). As in the general case of $\mathbb{X}$, the zero solution $\mathbf{x}(t) \equiv \mathbf{0} \in \mathbb{R}^{2}$ of system (8) is said to be (global) attractive if for every solution $\mathbf{x}(t)$ of (8), we have $\|\mathbf{x}(t)\|_{\mathbb{R}^{2}} \rightarrow 0$ as $t \rightarrow 0$. On the asymptotic stability and attractivity for several kinds of linear systems in the case 
when $t \rightarrow \infty$, we refer reader to [7-10,12-14] and references therein.

We know that a plain curve $\Gamma_{\mathbf{x}}$ is a Jordan curve in $\mathbb{R}^{2}$ if there exists a continuous injection $\mathbf{x}=\mathbf{x}(t), \mathbf{x}:\left[0, t_{0}\right] \rightarrow$ $\mathbb{R}^{2}, t_{0}>0$, such that $\mathbf{x}\left(\left[0, t_{0}\right]\right)=\Gamma_{\mathbf{x}}$. It is often said that $\Gamma_{\mathbf{x}}$ is parametrized by $\mathbf{x}(t)$ or that $\Gamma_{\mathbf{x}}$ is associated to $\mathbf{x}(t)$. The length of $\Gamma_{\mathbf{x}}$, denoted by length $\left(\Gamma_{\mathbf{x}}\right)$, is defined by

$$
\text { length }\left(\Gamma_{\mathbf{x}}\right)=\sup \sum_{k=1}^{m}\left\|\mathbf{x}\left(t_{k}\right)-\mathbf{x}\left(t_{k-1}\right)\right\|_{\mathbb{R}^{2}},
$$

where the supremum is taken over all finite partitions $0=$ $t_{m+1}<\cdots<t_{1}<t_{0}$ of the interval $\left[0, t_{0}\right]$ (see $\left.[17,18]\right)$.

Definition 4. The zero solution of system (8) is rectifiable attractive (resp., nonrectifiable attractive) if it is attractive and the curve $\Gamma_{\mathbf{x}}$ of every solution $\mathbf{x}$ of (8) is a rectifiable (resp., nonrectifiable) Jordan curve in $\mathbb{R}^{2}$.

Following [17], we are interested in Jordan curves so that the parametrization of our solutions faithfully represents the length and rectifiability properties (omitting injectivity, we might find solutions that self-intersect on large sets or are just self-winding and, hence, artificially nonrectifiable).

The following well-known fact (see [19]) gives us a main support to relate the singularity in (1) with the nonrectifiability in system (8):

$$
\left\|\frac{d \mathbf{x}}{d t}\right\|_{\mathbb{R}^{2}} \in L^{1}\left(0, t_{0}\right) \text { if and only if length }\left(\Gamma_{\mathbf{x}}\right)<\infty \text {. }
$$

On the rectifiability of graph of solutions of scalar secondorder linear differential equations, we refer reader to [20].

Here the particular attention is paid to the case of the socalled linear integrable systems defined as follows.

Definition 5. Let $\mathscr{M}_{2}$ denote the linear space of all $2 \times 2$ matrix with the elements in $\mathbb{C}$. We say that (8) is a linear integrable system if there exists an invertible matrix $T \in \mathscr{M}_{2}$ such that for every $t \in\left(0, t_{0}\right]$ the matrix $\Lambda(t)=T^{-1} A(t) T \in \mathscr{M}_{2}$ is a diagonal matrix for every $t \in\left(0, t_{0}\right]$.

Note that the matrices $A(t)$ are themselves always real. As commented in Section 6, we proceed with presentation of our results and arguments in terms of the coefficients of such matrices.

Denoting the matrix elements of $A(t)$ by

$$
A(t)=\left[\begin{array}{ll}
e(t) & f(t) \\
g(t) & h(t)
\end{array}\right], \quad t \in\left(0, t_{0}\right],
$$

when we have $e(t) \equiv h(t)$ and $f(t) \equiv-g(t)$, we speak of integrable systems (see for instance [21]). In such a case, both eigenvalues $\lambda(t)=h(t) \pm g(t) i$ of the matrix $A(t)$ admit eigenvectors $( \pm i, 1)$ that do not depend on variable $t$. This motivates us to introduce Definition 5 which gives a more general notion of the integrable system than previous one. It is because (see Lemma 11 in Section 4) the integrable system (8) has the following form:

$$
A(t)=\left[\begin{array}{cc}
h(t)+\mu g(t) & -v g(t) \\
\rho g(t) & h(t)
\end{array}\right], \quad t \in\left(0, t_{0}\right],
$$

where $\mu, v, \rho \in \mathbb{R}$. For $\mu=0$ and $\nu=\rho=1$ in (8), we have $e(t) \equiv h(t)$ and $f(t) \equiv-g(t)$; so, the classic integrable system is a special case.

In Section 5, we show that the set of all matrix-valued functions $A=A(t)$ that satisfy Definition 5 make an algebra.

Furthermore, we show in Section 4 that if (8) is a linear integrable system, then the matrix-valued function $A(t)$ satisfies the required hypotheses $\left(H_{1}\right)-\left(H_{2}\right)$ in particular for

$$
\begin{gathered}
\omega(t)=|g(t)|+|h(t)|, \\
a(t)=e^{-\int_{t}^{t_{0}}(h(\tau)+(\mu / 2) g(\tau)) d \tau}, \quad t \in\left(0, t_{0}\right], \\
U(t)=T \operatorname{diag}\left[e^{-\int_{t}^{t_{0}} \lambda_{1}(\tau) d \tau}, e^{-\int_{t}^{t_{0}} \lambda_{2}(\tau) d \tau}\right] T^{-1}, \quad t \in\left(0, t_{0}\right],
\end{gathered}
$$

where $\lambda_{1}(t)$ and $\lambda_{2}(t)$ are two eigenvalues of $A(t)$ for each $t \epsilon$ $\left(0, t_{0}\right]$. We also show that the solution curve of every solution of the integrable system (8) is a Jordan curve if for every pair $s, t \in\left(0, t_{0}\right], s<t$, at least one of the statements

$$
\begin{aligned}
& \int_{s}^{t}\left(h(\tau)+\frac{\mu}{2} g(\tau)\right) d \tau \neq 0 \\
& \quad \text { or } \quad \frac{\sqrt{4 \nu \rho-\mu^{2}}}{4 \pi} \int_{s}^{t} g(\tau) d \tau \notin \mathbb{Z}
\end{aligned}
$$

holds true, where $4 v \rho-\mu^{2}>0$.

This implies the third main result of the paper.

Theorem 6. Let $\mu^{2}-4 v \rho<0$. One supposes (15) and

$$
\lim _{t \rightarrow 0} \int_{t}^{t_{0}}\left(h(\tau)+\frac{1}{2} \mu g(\tau)\right) d \tau=\infty .
$$

The zero solution of an integrable system (8) with $A(t)$ of form (12) is nonrectifiable (resp., rectifiable) attractive if and only if

$$
\begin{array}{r}
(|h(t)|+|g(t)|) e^{-\int_{t}^{t_{0}}(h(\tau)+(1 / 2) \mu g(\tau)) d \tau} \notin L^{1}\left(0, t_{0}\right) \\
\left(\text { resp., } \in L^{1}\left(0, t_{0}\right)\right) .
\end{array}
$$

Remark 7. Under the assumptions of Theorem 6, we conclude that if $g(t) \equiv 0$, then the zero solution of integrable system (8) is rectifiable attractive.

The proof of the previous theorem is given in Section 4 based on Theorem 3. As an important consequence of preceding Theorem 6 , we are able to study the following twoparametric model-system, which is singular at $t=0$ :

$$
x^{\prime}(t)=\frac{h_{0}}{t^{a}} x(t)-\frac{1}{t^{b}} y(t), y^{\prime}(t)=\frac{1}{t^{b}} x(t)+\frac{h_{0}}{t^{a}} y(t),
$$

where $t \in\left(0, t_{0}\right]$.

Corollary 8. Let $h_{0}>0$ and $b \in \mathbb{R}$. One has that:

(i) if $a=1$, the zero solution of (18) is rectifiable attractive provided $b<h_{0}+1$ and nonrectifiable attractive provided $b \geq h_{0}+1$; 
(ii) if $a>1$, then the zero solution of (18) is rectifiable attractive for all $h_{0}>0$ and $b \in \mathbb{R}$.

As we see, in the case of $a=1$, the rectifiable attractivity of (18) depends on the order of growth of the singular term $t^{-b}$ appearing in the antidiagonal coefficients of $A(t)$. The existence of solutions for the integrable systems (8) and (18) is guaranteed by their explicit forms given in Lemma 11.

\section{Proofs of Theorems 1,2 , and 3}

In this section, we study the existence and uniqueness of solutions to (1) as well as its attractivity of the zero solution.

In the case when the operator-valued function $A=A(t)$, $A:\left[0, t_{0}\right] \rightarrow L(\mathbb{X})$ is defined and continuous on the whole interval $\left[0, t_{0}\right]$, then the existence of a classic solution $\mathbf{x}=\mathbf{x}(t)$ is well known; see, for instance, [22, Chapter 3], [23, Chapter 2], and [24, Chapter 5.1]; when $A(t)$ are unbounded linear operators, we refer reader to [24, Chapter 5.4] and some selected papers from [25].

However, our operator-valued function $A=A(t), A$ : $\left(0, t_{0}\right] \rightarrow L(\mathbb{X})$, is mainly singular and not defined at $t=0$. By this reason, we cannot use the existence results and methods obtained and used on $\left[0, t_{0}\right]$. One way to understand the main difficulties because of the pressumed singular behaviour of $A(t)$ near $t=0$, we can make the reflexion from $t=0$ into $\tau=\infty$ by the transformation $\tau=1 / t$. Then, the existence of a solution of equation $\mathbf{x}^{\prime}=$ $A(t) \mathbf{x}, t \in\left(0, t_{0}\right], \mathbf{x}\left(t_{0}\right)=\mathbf{x}_{0}$, can be related with the existence of a global solution of equation $\widetilde{\mathbf{x}}^{\prime}=\widetilde{A}(\tau) \widetilde{\mathbf{x}}, \tau \in\left[\tau_{0}, \infty\right)$, where $\widetilde{A}(\tau)=-\tau^{-2} A\left(\tau^{-1}\right), \widetilde{\mathbf{x}}(\tau)=\mathbf{x}(1 / t), \tau_{0}=1 / t_{0}$, and $\widetilde{\mathbf{x}}\left(t_{0}\right)=\widetilde{\mathbf{x}}_{0}$. Since $\left[\tau_{0}, \infty\right)$ is an infinite interval, our approach to the existence and uniqueness of solution of (1) is motivated by the works [26-29].

At first, it is clear that (1) can be rewritten in the form of corresponding integral operators equation:

$$
T \mathbf{x}=\mathbf{x} \in C\left(\left(0, t_{0}\right] ; \mathbb{X}\right),
$$

where $T: C\left(\left(0, t_{0}\right] ; \mathbb{X}\right) \rightarrow C\left(\left(0, t_{0}\right] ; \mathbb{X}\right)$ is defined by

$$
(T \mathbf{x})(t)=\mathbf{x}\left(t_{0}\right)-\int_{t}^{t_{0}} A(s) \mathbf{x}(s) d s, \quad t \in\left(0, t_{0}\right] .
$$

The existence and uniqueness of solution of problem (19)-(20) will be explored by the following $\alpha$-contraction fixed point result in Fréchet spaces.

Definition 9. Let $\left(F,|\cdot|_{n}\right)$ be a Fréchet space. A mapping $T$ : $F \rightarrow F$ is said to be an $\alpha$-contraction on $F$ if for each $n \in \mathbb{N}$ there exists $\alpha_{n} \in[0,1)$ such that

$$
|T \mathbf{x}-T \mathbf{y}|_{n} \leq \alpha_{n}|\mathbf{x}-\mathbf{y}|_{n}, \quad \forall \mathbf{x}, \mathbf{y} \in F .
$$

In applications, the inequality (21) need not be satisfied for the family of seminorms $|\cdot|_{n}$, rather only for another family of seminorms $\|\cdot\|_{n}$ which is equivalent to $|\cdot|_{n}$. Hence, the key point of the following $\alpha$-contraction principle is that an operator $T$ has a fixed point provided (21) is satisfied in $\|\cdot\|_{n}$.
Lemma 10. Let $\left(F,|\cdot|_{n}\right)$ be a Fréchet space and $T: F \rightarrow F$ be an $\alpha$-contraction on $F$ with respect to a family of seminorms $\|\cdot\|_{n}$ equivalent to $|\cdot|_{n}$. Then, $T$ has a fixed point on $F$. $1.2]$.

The previous lemma is a particular case of [29, Theorem

Proof of Theorem 1. Let $F=C\left(\left(0, t_{0}\right] ; \mathbb{X}\right), \mathbf{x} \in F$, and

$$
|\mathbf{x}|_{n}=\sup \left\{\|\mathbf{x}(t)\|_{\mathbb{X}}: t \in\left[\frac{1}{n}, t_{0}\right]\right\}
$$

It is known that $|\cdot|_{n}$ is a family of seminorms on $F$ and $\left(F,|\cdot|_{n}\right)$ is a Fréchet space.

Let $T: F \rightarrow F$ be an operator defined in (20). Since $A(t)$ may be singular at $t=0$, it is easy to check that in general $T$ is not an $\alpha$-contraction with respect to the family of seminorms $|x|_{n}$ given in (22).

Next, let $\omega_{n}$ and $h_{n}$ be two sequences of real numbers determined by

$$
\begin{gathered}
\omega_{n}=\sup \left\{\|A(t)\|: t \in\left[\frac{1}{n}, t_{0}\right]\right\}, \quad n \in \mathbb{N}, \\
h_{n}>0 \quad \text { such that } h_{n} \geq \omega_{n}, \quad n \in \mathbb{N} .
\end{gathered}
$$

Let us remark that if $\|A(t)\|$ satisfies (3), then obviously $\omega_{n} \rightarrow$ $\infty$ as $n \rightarrow \infty$. In respect to these two sequences $\omega_{n}$ and $h_{n}$, we define

$$
\|\mathbf{x}\|_{n}=\max \left\{e^{-h_{n}\left(t_{0}-t\right)}\|\mathbf{x}(t)\|_{\mathbb{X}}: t \in\left[\frac{1}{n}, t_{0}\right]\right\} .
$$

Such sequence $\|\cdot\|_{n}$ is a family of seminorms $\|\cdot\|_{n}$ which are equivalent with respect to $|\cdot|_{n}$ because

$$
e^{-h_{n}\left(t_{0}-1 / n\right)}|\mathbf{x}|_{n} \leq\|\mathbf{x}\|_{n} \leq|\mathbf{x}|_{n}, \quad \forall n \in \mathbb{N}, \forall \mathbf{x} \in F .
$$

Next, according to (20) and (23), for $t \in\left[1 / n, t_{0}\right]$, we calculate

$$
\begin{aligned}
\|T \mathbf{x}(t)-T \mathbf{y}(t)\|_{\mathbb{X}} & =\left\|\int_{t}^{t_{0}} A(s)(\mathbf{x}(s)-\mathbf{y}(s)) d s\right\|_{\mathbb{X}} \\
& \leq \int_{t}^{t_{0}}\|A(s)\|\|\mathbf{x}(s)-\mathbf{y}(s)\|_{\mathbb{X}} d s \\
& \leq \omega_{n} \int_{t}^{t_{0}}\|\mathbf{x}(s)-\mathbf{y}(s)\|_{\mathbb{X}} d s .
\end{aligned}
$$

Multiplying previous inequality by $e^{-h_{n}\left(t_{0}-t\right)}$, where $h_{n}$ is defined in (24), we obtain

$$
\begin{aligned}
e^{-h_{n}\left(t_{0}-t\right)}\|T \mathbf{x}(t)-T \mathbf{y}(t)\|_{\mathbb{X}} \\
\leq \omega_{n} e^{-h_{n}\left(t_{0}-t\right)} \int_{t}^{t_{0}}\|\mathbf{x}(s)-\mathbf{y}(s)\|_{\mathbb{X}} d s \\
=\omega_{n} \int_{t}^{t_{0}} e^{-h_{n}(s-t)} e^{-h_{n}\left(t_{0}-s\right)}\|\mathbf{x}(s)-\mathbf{y}(s)\|_{\mathbb{X}} d s \\
\leq \omega_{n}\|\mathbf{x}-\mathbf{y}\|_{n} \int_{t}^{t_{0}} e^{-h_{n}(s-t)} d s \leq \frac{\omega_{n}}{h_{n}}\|\mathbf{x}-\mathbf{y}\|_{n} .
\end{aligned}
$$


Taking the maximum in the previous inequality over the interval $\left[1 / n, t_{0}\right]$ and using (24) and (25), we conclude that

$$
\begin{aligned}
\|T \mathbf{x}-T \mathbf{y}\|_{n} \leq \alpha_{n}\|\mathbf{x}-\mathbf{y}\|_{n}, & \alpha_{n}=\frac{\omega_{n}}{h_{n}} \in[0,1), \\
& \forall n \in \mathbb{N}, \forall \mathbf{x}, \mathbf{y} \in F .
\end{aligned}
$$

Now, applying Lemma 10, we obtain the existence of $\mathbf{x} \in F$ satisfying problem (19)-(20). Since $\mathbf{x} \in F=C\left(\left(0, t_{0}\right] ; \mathbb{X}\right)$ and $A \in C\left(\left(0, t_{0}\right] ; L(\mathbb{X})\right)$, from (19)-(20) follows $\mathbf{x} \in C^{1}\left(\left(0, t_{0}\right] ; \mathbb{X}\right)$. Hence, by differentiating (19), we conclude that $\mathbf{x}=\mathbf{x}(t)$ is also a solution of equation (1). Thus, the existence of a solution of (1) is proved.

Now, we will show the uniqueness of solution of equation (1). Let $\mathbf{u}(t)$ and $\mathbf{v}(t)$ be two solutions of (1) such that $\mathbf{u}\left(t_{0}\right)=$ $\mathbf{v}\left(t_{0}\right)=\mathbf{x}_{0}$. Integrating (1) with respect to $\mathbf{u}(t)$ and $\mathbf{v}(t)$, we obtain $\mathbf{u}=T \mathbf{u}$ and $\mathbf{v}=T \mathbf{v}$, which yields $\|T \mathbf{u}-T \mathbf{v}\|_{n}=$ $\|\mathbf{u}-\mathbf{v}\|_{n}$. Puting this equality into (29), we conclude that

$$
\|\mathbf{u}-\mathbf{v}\|_{n} \leq \alpha_{n}\|\mathbf{u}-\mathbf{u}\|_{n}, \quad \alpha_{n}=\frac{\omega_{n}}{h_{n}} \in[0,1), \quad \forall n \in \mathbb{N} .
$$

On the other hand, if the solutions $\mathbf{u}(t)$ and $\mathbf{v}(t)$ are different, then there is $t^{*} \in\left(0, t_{0}\right]$ such that $\mathbf{u}\left(t^{*}\right) \neq \mathbf{v}\left(t^{*}\right)$, which implies that $\left\|\mathbf{u}\left(t^{*}\right)-\mathbf{v}\left(t^{*}\right)\right\|_{\mathbb{X}}>0$. In particular, there is a big enough $m \in \mathbb{N}$ such that $t^{*} \in\left[1 / m, t_{0}\right]$, and, hence,

$$
\begin{aligned}
\|\mathbf{u}-\mathbf{v}\|_{m} & =\max \left\{e^{-h_{m}\left(t_{0}-t\right)}\|\mathbf{u}-\mathbf{v}\|_{\mathbb{X}}: t \in\left[\frac{1}{m}, t_{0}\right]\right\} \\
& \geq e^{-h_{m}\left(t_{0}-t^{*}\right)}\left\|\mathbf{u}\left(t^{*}\right)-\mathbf{v}\left(t^{*}\right)\right\|_{\mathbb{X}}>0
\end{aligned}
$$

that is,

$$
\|\mathbf{u}-\mathbf{v}\|_{m}>0
$$

Putting (32) into (30), we obtain that $\alpha_{m} \geq 1$. But it is not possible since $\alpha_{m} \in[0,1)$. Hence, the assumption about the existence of $t^{*} \in\left(0, t_{0}\right]$ such that $\mathbf{u}\left(t^{*}\right) \neq \mathbf{v}\left(t^{*}\right)$ is not possible. Therefore, we conclude that $\mathbf{u}(t)=\mathbf{v}(t)$ for all $t \in\left(0, t_{0}\right]$, and, thus, the uniqueness of solution of (1) is shown.

Proof of Theorem 2. It is enough to show that if the statement (3) does not hold, then $\|d \mathbf{x} / d t\|_{\mathbb{X}} \in L^{1}\left(0, t_{0}\right)$ for every $\mathbf{x}_{0} \in \mathbb{X}$ and the corresponding solution $\mathbf{x}$ of (1) such that $\mathbf{x}\left(t_{0}\right)=\mathbf{x}_{0}$. In fact, if we suppose contrary to (3), then there exists $c_{0}>0$ such that $\|A(t)\| \leq c_{0}, t \in\left(0, t_{0}\right]$. Also, because of $\|\mathbf{x}(t)\|_{\mathbb{X}} \rightarrow$ 0 as $t \rightarrow 0$, there exists $c_{1}>0$ such that $\|\mathbf{x}(t)\|_{\mathbb{X}} \leq c_{1}, t \in$ $\left(0, t_{0}\right]$. Hence, from $(1)$, we obtain

$$
\begin{array}{r}
\left\|\frac{d \mathbf{x}}{d t}\right\|_{\mathbb{X}}=\|A(t) \mathbf{x}(t)\|_{\mathbb{X}} \leq\|A(t)\|\|\mathbf{x}(t)\|_{\mathbb{X}} \leq c_{0} c_{1}, \\
t \in\left(0, t_{0}\right],
\end{array}
$$

which shows that $\|d \mathbf{x} / d t\|_{\mathbb{X}} \in L^{1}\left(0, t_{0}\right)$.

Proof of Theorem 3. The conclusion (i) immediately follows from the hypothesis $\left(H_{2}\right)$ because $\|\mathbf{x}(t)\|_{\mathbb{X}}=\left\|U(t) \mathbf{x}_{0}\right\|_{\mathbb{X}} \leq$ $\|U(t)\|\left\|\mathbf{x}_{0}\right\|_{\mathbb{X}} \rightarrow 0$ as $t \rightarrow 0$.
Next, we give the proof of the conclusion (ii). Let $s, t \in$ $\left(0, t_{0}\right], s<t$, be such that $\mathbf{x}_{0}$ is an eigenvector of $U^{-1}(s) U(t)$ with an eigenvalue 1 . Then,

$$
U^{-1}(s) U(t) \mathbf{x}_{0}=\mathbf{x}_{0} .
$$

Applying $U(s)$ to the pervious formula, we obtain the expression $U(t) \mathbf{x}_{0}=U(s) \mathbf{x}_{0}$, which by definition of $\mathbf{x}(t)$ equals to $\mathbf{x}(t)=\mathbf{x}(s)$. Hence, $\mathbf{x}=\mathbf{x}(t)$ is not an injective function. Note that, since $U(t)$ is postulated invertible for all $t \in\left(0, t_{0}\right]$, all the steps of this proof are reversible. Thus, the required equivalence holds.

Finally, the conclusion (iii) follows from the hypotheses $\left(H_{1}\right)-\left(H_{2}\right)$ because

$$
\begin{aligned}
c_{0} c_{2} a(t) \omega(t)\left\|\mathbf{x}_{0}\right\|_{\mathbb{X}} & \leq c_{0} \omega(t)\left\|U(t) \mathbf{x}_{0}\right\|_{\mathbb{X}}=c_{0} \omega(t)\|\mathbf{x}(t)\|_{\mathbb{X}} \\
& \leq\|A(t) \mathbf{x}(t)\|_{\mathbb{X}}, \\
\|A(t) \mathbf{x}(t)\|_{\mathbb{X}} & \leq c_{1} \omega(t)\|\mathbf{x}(t)\|_{\mathbb{X}}=c_{1} \omega(t)\left\|U(t) \mathbf{x}_{0}\right\|_{\mathbb{X}} \\
& \leq c_{1} c_{3} a(t) \omega(t)\left\|\mathbf{x}_{0}\right\|_{\mathbb{X}} .
\end{aligned}
$$

Hence, the required equivalence in (iii) holds.

Note that conclusion (ii) does not depend on any of the hypotheses $\left(H_{1}\right)-\left(H_{2}\right)$.

\section{Proofs of Theorem 6 and Corollary 8}

In this section, we study the rectifiable and nonrectifiable attractivity of the linear integrable systems (8).

At the first, we state the following lemma in which a specific form of the matrix $A(t)$ of linear integrable systems is proposed.

Lemma 11. Suppose that system (8) is integrable. Then, there exist functions $g, h:\left(0, t_{0}\right] \rightarrow \mathbb{R}$ and constants $\mu, \nu, \rho \in \mathbb{R}$ such that

$$
A(t)=\left[\begin{array}{cc}
h(t)+\mu g(t) & -v g(t) \\
\rho g(t) & h(t)
\end{array}\right], \quad t \in\left(0, t_{0}\right] .
$$

The eigenvalues $\lambda_{1,2}:\left(0, t_{0}\right] \rightarrow \mathbb{C}$ of $A(t)$ are given by the formula

$$
\lambda_{1,2}(t)=\left(h(t)+\frac{\mu}{2} g(t)\right) \pm|g(t)| \sqrt{\frac{\mu^{2}}{4}-\rho \nu} .
$$

Proof. Let $\lambda_{1,2}:\left(0, t_{0}\right] \rightarrow \mathbb{C}$ be two eigenvalues of the matrix-valued function $A(t)$. Since system (8) is supposed to be an integrable system, from Definition 5 , there is a regular matrix $T \in \mathscr{M}_{2}$ such that

$$
A(t)=T \operatorname{diag}\left[\lambda_{1}(t), \lambda_{2}(t)\right] T^{-1}, \quad t \in\left(0, t_{0}\right]
$$

If $\operatorname{det}(T) \in \mathbb{R}$ and $\operatorname{det}(T)<0$, then we can change the columns in $T$ which causes $\operatorname{det}(T)>0$ and $\operatorname{diag}\left[\lambda_{2}(t), \lambda_{1}(t)\right]$ 
instead of $\operatorname{diag}\left[\lambda_{1}(t), \lambda_{2}(t)\right]$. Also, if $\operatorname{det}(T) \in \mathbb{R}$ and $\operatorname{det}(T)>$ 0 or $\operatorname{det}(T) \in \mathbb{C} \backslash \mathbb{R}$, then

$$
\begin{aligned}
& A(t)=T_{0} \operatorname{diag}\left[\lambda_{2}(t), \lambda_{1}(t)\right] T_{0}^{-1}, \\
& \operatorname{det}\left(T_{0}\right)=1, \quad \text { where } T_{0}=\frac{1}{\sqrt{\operatorname{det}(T)}} T .
\end{aligned}
$$

Hence, we may suppose that $\operatorname{det}(T)=1$; that is, for some complex numbers $\alpha, \beta, \gamma, \delta$ with $\alpha \delta-\beta \gamma=1$, we may set

$$
T=\left[\begin{array}{ll}
\alpha & \beta \\
\gamma & \delta
\end{array}\right], \quad T^{-1}=\left[\begin{array}{cc}
\delta & -\beta \\
-\gamma & \alpha
\end{array}\right]
$$

Now from (38) and (40), we obtain

$$
A(t)=\left[\begin{array}{ll}
\alpha \delta \lambda_{1}(t)-\beta \gamma \lambda_{2}(t) & \alpha \beta\left(\lambda_{2}(t)-\lambda_{1}(t)\right) \\
\gamma \delta\left(\lambda_{1}(t)-\lambda_{2}(t)\right) & \alpha \delta \lambda_{2}(t)-\beta \gamma \lambda_{1}(t)
\end{array}\right],
$$

where the matrix elements of $A(t)$ are real numbers for all $t \in\left(0, t_{0}\right]$.

We denote that

$$
h(t):=\alpha \delta \lambda_{2}(t)-\beta \gamma \lambda_{1}(t), \quad t \in\left(0, t_{0}\right]
$$

and if $\lambda_{1,2}(t) \in \mathbb{R}$ for all $t \in\left(0, t_{0}\right]$, then let

$$
\begin{aligned}
& g(t):=\lambda_{1}(t)-\lambda_{2}(t), \quad \rho:=\gamma \delta, \\
& \nu:=\alpha \beta, \quad \mu:=-(\alpha \delta+\gamma \beta),
\end{aligned}
$$

and if $\lambda_{1,2}(t)$ form a complex-conjugate pair for all $t \in\left(0, t_{0}\right]$, then let

$$
\begin{aligned}
& g(t):=-i\left(\lambda_{1}(t)-\lambda_{2}(t)\right), \quad \rho:=i \gamma \delta, \\
& \nu:=i \alpha \beta, \quad \mu:=-i(\alpha \delta+\gamma \beta) .
\end{aligned}
$$

Putting previous notations in (41), we get the desired conclusion (36). Note that all of these functions and constants are real, since $h$ is defined as a matrix-element of a real matrix, $g$ is defined real in both cases, and the rest of the constants are necessarily real by virtue of satisfying (36). Now, the conclusion (37) immediately follows from (36).

By Theorem 1, we know that there exists a unique solution $\mathbf{x}(t)$ of system (8). Moreover, in the next lemma, we show that if (8) is a linear integrable system, then the solution $\mathbf{x}(t)$ is explicitly expressed by the matrix elements of $A(t)$.

Lemma 12. Let $\mathbf{x}$ be a solution of linear integrable system (8) and let $T \in \mathscr{M}_{2}$ be a matrix determined in Definition 5. Then, there exists a matrix-valued function $U=U(t), U$ : $\left(0, t_{0}\right] \rightarrow \mathscr{M}_{2}$, such that $\mathbf{x}(t)=U(t) \mathbf{x}_{0}$ and satisfies the following properties:

(1) $U\left(t_{0}\right)$ is the identity matrix;

(2) $U(t)$ is invertible for every $t \in\left(0, t_{0}\right]$;

(3) $T^{-1} U(t) T$ is a diagonal matrix for every $t \in\left(0, t_{0}\right]$;
(4) if $\lambda(t)$ is an eigenvalue of $A(t)$, then $e^{-\int_{t}^{t_{0}} \lambda(\tau) d \tau}$ is an eigenvalue of $U(t)$;

(5) $U^{\prime}(t)=A(t) U(t)$ for every $t \in\left(0, t_{0}\right]$.

Proof. Let $T$ be the matrix that diagonalizes $A(t)$. Let $\Lambda_{A}(t)=$ $T^{-1} A(t) T$ be of form

$$
\Lambda_{A}(t)=\left[\begin{array}{cc}
\lambda_{1}(t) & 0 \\
0 & \lambda_{2}(t)
\end{array}\right]
$$

We define

$$
\begin{aligned}
& \Lambda_{U}(t)=\left[\begin{array}{cc}
e^{-\int_{t}^{t_{0}} \lambda_{1}(\tau) d \tau} & 0 \\
0 & e^{-\int_{t}^{t_{0}} \lambda_{2}(\tau) d \tau}
\end{array}\right], \\
& U(t)=T \Lambda_{U}(t) T^{-1} .
\end{aligned}
$$

Now, $\Lambda_{U}\left(t_{0}\right)$ is obviously the identity matrix, and thus $U\left(t_{0}\right)=T I T^{-1}=I$. Since $\Lambda_{U}(t)$ is invertible for all $t \epsilon$ $\left(0, t_{0}\right]$, so is $U(t)$. Next, $T^{-1} U(t) T=\Lambda_{U}(t)$ is diagonal by construction. For an eigenvalue $\lambda(t)$ of $A(t)$, by construction, $\exp \left\{-\int_{t}^{t_{0}} \lambda(\tau) d \tau\right\}$ is a diagonal element of $\Lambda_{U}(t)$; so, it is an eigenvalue of $U(t)$. Also,

$$
\begin{aligned}
U^{\prime}(t) & =T \Lambda_{U}^{\prime}(t) T^{-1}=T \Lambda_{A}(t) \Lambda_{U}(t) T^{-1} \\
& =T \Lambda_{A}(t) T^{-1} T \Lambda_{U}(t) T^{-1}=A(t) U(t) .
\end{aligned}
$$

And finally, the function $\mathbf{x}(t)=U(t) \mathbf{x}_{0}$ solves the integrable system (8) because

$$
\mathbf{x}^{\prime}(t)=U^{\prime}(t) \mathbf{x}_{0}=A(t) U(t) \mathbf{x}_{0}=A(t) \mathbf{x}(t)
$$

and has the property $\mathbf{x}\left(t_{0}\right)=U\left(t_{0}\right) \mathbf{x}_{0}=\mathbf{x}_{0}$.

According to [23, p. 188], the matrix-valued function $U(t)$ is called the evolution operator.

In the next lemma, we give some necessary and sufficient conditions such that the solution's curve $\Gamma_{\mathbf{x}}$ of every solution $\mathbf{x}(t)$ of integrable system (8) is a Jordan curve. Results of that kind are important because Definition 4 requires the Jordan property.

Lemma 13. Let (8) be a linear integrable system such that $\mu^{2}-$ $4 v \rho<0$ and $g(t) \neq 0, t \in\left(0, t_{0}\right]$, where the real numbers $\mu, v$, $\rho$, and $g(t)$ are determined by Lemma 11. Then, the solution's curve $\Gamma_{\mathbf{x}}$ of every solution $\mathbf{x}(t)$ is a Jordan curve in $\mathbb{R}^{2}$ if and only if for every pair $s, t \in\left(0, t_{0}\right], s<t$, at least one of the statements in (15) holds true.

Proof. In order to prove this lemma, we use the equivalence stated in the conclusion (ii) of Theorem 3 (we may use Theorem 3 because of Lemma 12; see also the remark at the end of Theorem 3). According to that, we need to find pairs $s$ and $t$ such that 1 is an eigenvalue of $U^{-1}(s) U(t)$. Since the evolution operator $U(t)$ can be diagonalized into the form $\Lambda_{U}(t)$, we compute

$$
\begin{aligned}
U^{-1}(s) U(t) & =\left(T \Lambda_{U}(s) T^{-1}\right)^{-1} T \Lambda_{U}(t) T^{-1} \\
& =T \Lambda_{U}^{-1}(s) \Lambda_{U}(t) T^{-1} .
\end{aligned}
$$


The diagonalized form of $U^{-1}(s) U(t)$ is then given by the formula

$$
\begin{aligned}
\Lambda_{U}^{-1} & (s) \Lambda_{U}(t) \\
= & {\left[\begin{array}{cc}
e^{\int_{s}^{t_{0}} \lambda_{1}(\tau) d \tau} & 0 \\
0 & e^{\int_{s}^{t_{0}}} \lambda_{2}(\tau) d \tau
\end{array}\right]\left[\begin{array}{cc}
e^{-\int_{t}^{t_{0}} \lambda_{1}(\tau) d \tau} & 0 \\
0 & e^{-\int_{t}^{t_{0}} \lambda_{2}(\tau) d \tau}
\end{array}\right] } \\
= & {\left[\begin{array}{cc}
e^{-\int_{t}^{s} \lambda_{1}(\tau) d \tau} & 0 \\
0 & e^{-\int_{t}^{s} \lambda_{2}(\tau) d \tau}
\end{array}\right] . }
\end{aligned}
$$

Now, the eigenvalues of $U^{-1}(s) U(t)$ are $e^{-\int_{t}^{s} \lambda_{1}(\tau) d \tau}$ and $e^{-\int_{t}^{s} \lambda_{2}(\tau) d \tau}$. Since $\lambda_{2}=\overline{\lambda_{1}}$, we only need to solve the equation $e^{-\int_{t}^{s} \lambda_{1}(\tau) d \tau}$

$$
\begin{aligned}
=e^{-\int_{t}^{s}(h(\tau)+(\mu / 2) g(\tau) d \tau)}\left(\cos \left(\frac{1}{2} \sqrt{4 \nu \rho-\mu^{2}} \int_{t}^{s} g(\tau) d \tau\right)\right. \\
+i \sin \left(\frac{1}{2} \sqrt{4 \nu \rho-\mu^{2}}\right. \\
\left.\left.\times \int_{t}^{s} g(\tau) d \tau\right)\right)=1 .
\end{aligned}
$$

This equation is solved whenever

$$
\begin{gathered}
\int_{s}^{t}\left(h(\tau)+\frac{\mu}{2} g(\tau) d \tau\right)=0, \\
\frac{1}{2} \sqrt{4 \nu \rho-\mu^{2}} \int_{s}^{t} g(\tau) d \tau=2 k \pi, \quad \text { for some } k \in \mathbb{Z},
\end{gathered}
$$

where the order of integration has been reversed by multiplying by -1 .

Since by this argument, $U^{-1}(s) U(t)=I$ if and only if 1 is an eigenvalue of $U^{-1}(s) U(t)$, we get the full equivalence.

Proof of Theorem 6. The key point of this proof is to show that the integrable system (8) satisfies the required hypotheses $\left(H_{1}\right)-\left(H_{2}\right)$, because all conclusions of Theorem 6 follow immediately from the conclusions (i), (ii), and (iii) of Theorem 3. Before we show that, we state the following two propositions which will be proved in Section 5 .

Proposition 14. Let $f: \mathbb{R} \times \mathbb{R} \rightarrow \mathbb{R}$ be defined by $f(x, y)=$ $\sqrt{x^{2}+\mu x y+\rho v y^{2}}$, where $\mu, \nu, \rho$ are real numbers such that $\mu^{2}<4 \nu \rho$. Then, there exist constants $c_{1}, c_{2}>0$ such that

$$
c_{1}(|x|+|y|) \leq f(x, y) \leq c_{2}(|x|+|y|), \quad \forall x, y \in \mathbb{R} .
$$

Proposition 15. Let $\Lambda:\left(0, t_{0}\right] \rightarrow \mathscr{M}_{2}$ be a diagonal matrix of the form

$$
\Lambda(t)=\left[\begin{array}{cc}
\lambda(t) & 0 \\
0 & \bar{\lambda}(t)
\end{array}\right],
$$

for all $t \in\left(0, t_{0}\right]$. Then, $\operatorname{det} \Lambda(t) \geq 0, t \in\left(0, t_{0}\right]$ and

$$
\begin{array}{r}
\|\Lambda(t) \vec{z}\|=|\lambda(t)|\|\vec{z}\|=\sqrt{\operatorname{det} \Lambda(t)}\|\vec{z}\| \\
\forall t \in\left(0, t_{0}\right], \vec{z} \in \mathbb{C}^{2} .
\end{array}
$$

Now we proceed with the proof of Theorem 6. By definition, $A(t)=T^{-1} \Lambda_{A}(t) T$, where $T \in \mathscr{M}_{2}$ is an invertible matrix and $\Lambda_{A}:\left(0, t_{0}\right] \rightarrow \mathscr{M}_{2}$ is a matrix-valued function given in (45). Since $T$ is invertible, there exist constants $c_{1}, c_{2}>0$ such that

$$
\begin{gathered}
c_{1}\|\vec{z}\| \leq\|T \vec{z}\| \leq c_{2}\|\vec{z}\|, \quad \forall \vec{z} \in \mathbb{C}^{2}, \\
\frac{1}{c_{2}}\|\vec{w}\| \leq\left\|T^{-1} \vec{w}\right\| \leq \frac{1}{c_{1}}\|\vec{w}\|, \quad \forall \vec{w} \in \mathbb{C}^{2} .
\end{gathered}
$$

Next, from (36), we get

$$
\begin{aligned}
\sqrt{\operatorname{det} \Lambda_{A}(t)} & =\sqrt{\operatorname{det} A(t)} \\
& =\sqrt{h^{2}(t)+\mu g(t) h(t)+\rho v g^{2}(t)} \\
& =f(h(t), g(t)), \quad t \in\left(0, t_{0}\right],
\end{aligned}
$$

where $f(x, y)$ is defined in Proposition 14 . Hence, by (57) and Propositions 14 and 15 applied to $\Lambda(t)=\Lambda_{A}(t)$, we get two constants $c_{3}, c_{4}>0$ such that

$$
c_{3} \omega(t)\|\vec{z}\| \leq\left\|\Lambda_{A}(t) \vec{z}\right\| \leq c_{4} \omega(t)\|\vec{z}\|, \quad t \in\left(0, t_{0}\right], \vec{z} \in \mathbb{C}^{2},
$$

where $\omega(t)=|h(t)|+|g(t)|$. Now from (56) and (58), for all $t \in\left(0, t_{0}\right]$ and $\vec{x} \in \mathbb{R}^{2}$, we have

$$
\begin{aligned}
\frac{c_{1} c_{3}}{c_{2}} \omega(t)\|\vec{x}\| & \leq \frac{c_{3}}{c_{2}} \omega(t)\|T \vec{x}\| \\
& \leq \frac{1}{c_{2}}\left\|\Lambda_{A}(t) T \vec{x}\right\| \leq\left\|T^{-1} \Lambda_{A}(t) T \vec{x}\right\| \\
& =\|A(t) \vec{x}\|, \\
\|A(t) \vec{x}\| & =\left\|T^{-1} \Lambda_{A}(t) T \vec{x}\right\| \leq \frac{1}{c_{1}}\left\|\Lambda_{A}(t) T \vec{x}\right\| \\
& \leq \frac{c_{4}}{c_{1}} \omega(t)\|T \vec{x}\| \leq \frac{c_{4} c_{2}}{c_{1}} \omega(t)\|\vec{x}\|,
\end{aligned}
$$

which shows that the hypothesis $\left(H_{1}\right)$ is fulfilled with respect to $\omega(t)$ given in (13).

Next, let the evolution operator $U(t)$ and matrix-valued function $\Lambda_{U}:\left(0, t_{0}\right] \rightarrow \mathscr{M}_{2}$ be defined by (46). The required condition $\left(\mathrm{H}_{2}\right)$ is satisfied because of the following arguments. First, from Lemma 12 and (37), we have

$$
\begin{array}{r}
\sqrt{\operatorname{det} \Lambda_{U}(t)}=\sqrt{\operatorname{det} U(t)}=e^{-\int_{t}^{t_{0}}(h(\tau)+(\mu / 2) g(\tau)) d \tau}=: a(t), \\
t \in\left(0, t_{0}\right] .
\end{array}
$$


Hence, by Proposition 15 applied to $\Lambda(t)=\Lambda_{U}(t)$, we obtain

$$
\left\|\Lambda_{U}(t) \vec{z}\right\|=a(t)\|\vec{z}\|, \quad t \in\left(0, t_{0}\right], \vec{z} \in \mathbb{C}^{2} .
$$

Now from (56) and (61), for all $t \in\left(0, t_{0}\right]$ and $x \in \mathbb{R}^{2}$, we have

$$
\begin{aligned}
\frac{c_{1}}{c_{2}} a(t)\|\vec{x}\| & \leq \frac{1}{c_{2}} a(t)\|T \vec{x}\|=\frac{1}{c_{2}}\left\|\Lambda_{U}(t) T \vec{x}\right\| \\
& \leq\left\|T^{-1} \Lambda_{U}(t) T \vec{x}\right\|=\|U(t) \vec{x}\|, \\
\|U(t) \vec{x}\| & =\left\|T^{-1} \Lambda_{U}(t) T \vec{x}\right\| \leq \frac{1}{c_{1}}\left\|\Lambda_{U}(t) T \vec{x}\right\| \\
& =\frac{1}{c_{1}} a(t)\|T \vec{x}\| \leq \frac{c_{2}}{c_{1}} a(t)\|\vec{x}\|,
\end{aligned}
$$

which shows that $U(t)$ satisfied the required inequalities in $\left(H_{2}\right)$ with respect to $a(t)$ given in (13).

Finally, by Lemma 13, we obtain that the solution's curve $\Gamma_{\mathbf{x}}$ of every solution $\mathbf{x}$ is a Jordan curve. Thus, all assumptions of Theorem 3 are fulfilled, and, therefore, we may apply Theorem 3 here. Consequently, the proof of this theorem is complete.

Proof of Corollary 8. It is enough to show that all assumptions of Theorem 6: $\mu^{2}-4 v \rho<0,(15),(16)$, and (17) are fulfilled in particular for

$$
\begin{aligned}
& h(t)=h_{0} t^{-a}, \quad g(t)=t^{-b}, \\
& \mu=0, \quad v=\rho=1,
\end{aligned}
$$

where $h_{0}>0, a \geq 1$, and $b \in \mathbb{R}$. In fact, the following elementary calculation is shown:

$$
\begin{aligned}
\mu^{2}-4 \nu \rho & =-4<0, \\
\int_{s}^{t}\left(h(\tau)+\frac{\mu}{2} g(\tau)\right) d \tau & =\int_{s}^{t} h(\tau) d \tau \\
& =h_{0} \int_{s}^{t} \tau^{-a} d \tau \neq 0, \quad s \neq t, \\
\lim _{t \rightarrow 0} \int_{t}^{t_{0}}\left(h(\tau)+\frac{1}{2} \mu g(\tau)\right) d \tau & =h_{0} \lim _{t \rightarrow 0} \int_{t}^{t_{0}} \tau^{-a} d \tau=\infty .
\end{aligned}
$$

Moreover, for $a=1$,

$$
\begin{aligned}
& (|h(t)|+|g(t)|) e^{-\int_{t}^{t_{0}}(h(\tau)+(1 / 2) \mu g(\tau)) d \tau} \\
& \quad=t_{0}^{-h_{0}}\left(h_{0} t^{-1+h_{0}}+t^{-b+h_{0}}\right) \notin L^{1}\left(0, t_{0}\right) ;
\end{aligned}
$$

so, condition (17) is fulfilled if and only if $b \geq h_{0}+1$. Also, for $a>1$,

$$
\begin{aligned}
& (|h(t)|+|g(t)|) e^{-\int_{t}^{t_{0}}(h(\tau)+(1 / 2) \mu g(\tau)) d \tau} \\
& \quad=\left(h_{0} t^{-a}+t^{-b}\right) e^{-\left(h_{0} /(a-1)\right)\left(t^{1-a}-t_{0}^{1-a}\right)} \in L^{1}\left(0, t_{0}\right) .
\end{aligned}
$$

Therefore, we may apply Theorem 6 to system (18) which proves this corollary.

\section{Appendix}

In this section, we first state a result saying that the set of all matrix-valued functions $A:\left(0, t_{0}\right] \rightarrow \mathscr{M}_{2}$ which satisfy Definition 5 forms an algebra. In this direction, let $\mathscr{A}\left(\left(0, t_{0}\right] ; \mathscr{M}_{2}\right)$ be a set defined by

$$
\begin{aligned}
& \mathscr{A}\left(\left(0, t_{0}\right] ; \mathscr{M}_{2}\right) \\
& =\left\{A:\left(0, t_{0}\right] \longrightarrow \mathscr{M}_{2} \mid \exists T \in \mathscr{M}_{2}, \operatorname{det} T \neq 0,\right. \\
& \left.\quad \text { such that } T^{-1} A(t) T \text { is diagonal, } \forall t \in\left(0, t_{0}\right]\right\} .
\end{aligned}
$$

Theorem 16. The set $\mathscr{A}\left(\left(0, t_{0}\right] ; \mathscr{M}_{2}\right)$ is an algebra with respect to the classic matrix operations + and $\circ$; that is to say, if $A, B \in$ $\mathscr{A}\left(\left(0, t_{0}\right] ; \mathscr{M}_{2}\right)$, then the following properties hold:

(1) $\alpha A+\beta B \in \mathscr{A}\left(\left(0, t_{0}\right] ; \mathscr{M}_{2}\right)$ for all $\alpha, \beta \in \mathbb{R}$;

(2) $A \circ B \in \mathscr{A}\left(\left(0, t_{0}\right] ; \mathscr{M}_{2}\right)$;

(3) if $\operatorname{det} A(t) \neq 0$ for all $t \in\left(0, t_{0}\right]$, then $A^{-1} \in$ $\mathscr{A}\left(\left(0, t_{0}\right] ; \mathscr{M}_{2}\right)$;

(4) if $f: \mathbb{R} \rightarrow \mathbb{R}$ is a real analytic function such that $f(A(t))$ converges absolutely for all $t \in J$, then $f(A) \in$ $\mathscr{A}\left(\left(0, t_{0}\right] ; \mathscr{M}_{2}\right)$.

Proof. We denote the diagonal matrices $\Lambda_{A}=T A T^{1}$ and $\Lambda_{B}=T B T^{1}$.

(1) $T(\alpha A+\beta B) T^{-1}=\alpha T A T^{-1}+\beta T B T^{-1}=\alpha \Lambda_{A}+\beta \Lambda_{B}$, which is diagonal.

(2) $T_{A B T^{-1}}=T A T^{-1} T B T^{-1}=\Lambda_{A} \Lambda_{B}$, which is diagonal.

(3) $\left(T A^{-1} T^{-1}\right)=\left(T A T^{-1}\right)^{-1}=\Lambda_{A}^{-1}$, which is also diagonal.

(4) $T f(A) T^{-1}=T\left(\sum_{n} a_{n} A^{n}\right) T^{-1}=\sum_{n} a_{n} T A^{n} T^{-1}=$ $\sum_{n} a_{n} \Lambda_{A}^{n}$, which is also diagonal.

Next, we give the proofs of some technical results used in previous sections for proving the main results.

Proof of Proposition 14. Denote by $u \in \mathbb{R}^{2}$ the vector $u=$ $(x, y)$. It is enough to show that $f=f(u)$ is a norm as a function $f: \mathbb{R}^{2} \rightarrow \mathbb{R}$, since all the norms in $\mathbb{R}^{2}$ are equivalent [30, p. 38], which, by definition, is the claim of this lemma. Let

$$
M=\left[\begin{array}{cc}
1 & \frac{\mu}{2} \\
\frac{\mu}{2} & \rho \nu
\end{array}\right] .
$$

By direct computation, we see that $f(u)=\sqrt{(M u, u)}$. Since $M$ is symmetric and positive definite, it has a unique symmetric positive definite square root $N^{2}=M$, so that $f(u)=$ $\sqrt{(N u, N u)}$ [31, p. 231]. Hence, $f(u)=\|v\|_{\mathbb{R}^{2}}$, where $v=N u$; so, the function $f=f(u)$ is a norm. 
Proof of Proposition 15. Let $\phi(t):\left(0, t_{0}\right] \rightarrow \mathbb{R}$ be such that $\lambda(t)=|\lambda(t)| e^{i \phi(t)}$. Then, $\bar{\lambda}(t)=|\lambda(t)| e^{-i \phi(t)}$; so, for any $z=$ $\left(z_{1}, z_{2}\right) \in \mathbb{C}^{2}$, we have

$$
\begin{aligned}
\|\Lambda(t) z\| & =|\lambda(t)|\left\|\left(e^{i \phi(t)} z_{1}, e^{-i \phi(t)} z_{2}\right)\right\| \\
& =|\lambda(t)| \sqrt{\left|e^{i \phi(t)} z_{1}\right|^{2}+\left|e^{-i \phi(t)} z_{2}\right|^{2}}=|\lambda(t)|\|z\|,
\end{aligned}
$$

since $\left|e^{ \pm i \phi}\right|=1$. The remaining equality is true because

$$
|\lambda(t)|=\sqrt{\lambda(t) \overline{\lambda(t)}}=\sqrt{\operatorname{det} \Lambda(t)} .
$$

\section{Final Remarks}

In our approach to the model over $\mathbb{R}^{2}$, we have chosen to emphasize the a priori structure of the real matrices describing the integrable systems. Thus, we have stated and proved this theory in terms of such real functions, complexifying only when absolutely necessary.

We could have taken another approach, complexifying from the start and then easily diagonalizing all the relevant matrices, but this would not essentially simplify any argument except in notation, while we would run the risk of confusing the reader even further about which term or function is real and which one is complex.

\section{References}

[1] F. Amato, M. Ariola, M. Carbone, and C. Cosentino, "Finitetime control of linear systems: a survey," in Current Trends in Nonlinear Systems and Control, Systems \& Control: Foundations \& Applications, pp. 195-213, Birkhäuser, Boston, Mass, USA, 2006.

[2] G. Haller, "A variational theory of hyperbolic Lagrangian coherent structures," Physica D, vol. 240, no. 7, pp. 574-598, 2011.

[3] T. Peacock and J. Dabiri, "Introduction to focus issue: lagrangian coherent structures," Chaos, vol. 20, no. 1, Article ID 017501, 2010.

[4] K. Rateitschak and O. Wolkenhauer, "Thresholds in transient dynamics of signal transduction pathways," Journal of Theoretical Biology, vol. 264, no. 2, pp. 334-346, 2010.

[5] I. Rachůnková and L. Rachůnek, "Asymptotic formula for oscillatory solutions of some singular nonlinear differential equation," Abstract and Applied Analysis, vol. 2011, Article ID 981401, 9 pages, 2011.

[6] B. B. Singh and I. M. Chandarki, "On the asymptotic behaviours of solutions of third order non-linear autonomous differential equation governing the MHD flow, Differential Equations \& Applications, vol. 3, no. 3, pp. 385-397, 2011.

[7] P. Hartman, Ordinary Differential Equations, Birkhäauser, Boston, Mass, USA, 2nd edition, 1982.

[8] W. A. Coppel, Stability and Asymptotic Behavior of Differential Equations, D. C. Heath and Co., Boston, Mass, USA, 1965.

[9] S. Castillo and M. Pinto, "Asymptotic integration of ordinary different systems," Journal of Mathematical Analysis and Applications, vol. 218, no. 1, pp. 1-12, 1998.
[10] S. Bodine, "A dynamical systems result on asymptotic integration of linear differential systems," Journal of Differential Equations, vol. 187, no. 1, pp. 1-22, 2003.

[11] R. Medina and M. Pinto, "On the asymptotic behavior of solutions of certain second order nonlinear differential equations," Journal of Mathematical Analysis and Applications, vol. 135, no. 2, pp. 399-405, 1988.

[12] H. Matsunaga, "Stability switches in a system of linear differential equations with diagonal delay," Applied Mathematics and Computation, vol. 212, no. 1, pp. 145-152, 2009.

[13] S. K. Choi and N. J. Koo, "Asymptotic property for linear integro-differential systems," Nonlinear Analysis: Theory, Methods \& Applications, vol. 70, no. 5, pp. 1862-1872, 2009.

[14] S. K. Choi, N. J. Koo, and S. Dontha, "Asymptotic property in variation for nonlinear differential systems," Applied Mathematics Letters, vol. 18, no. 1, pp. 117-126, 2005.

[15] C. Qian and Y. Sun, "Global attractivity of solutions of nonlinear delay differential equations with a forcing term," Nonlinear Analysis: Theory, Methods \& Applications, vol. 66, no. 3, pp. 689703, 2007.

[16] S. Miličić, Geometric and fractal properties of solutions of linear differential systems [Ph.D. thesis], University of Zagreb, Zagreb, Croatia.

[17] K. Falconer, Fractal Geometry: Mathematical Fondations and Applications, John Wiley \& Sons, New York, NY, USA, 1999.

[18] C. Tricot, Curves and Fractal Dimension, Springer, New York, NY, USA, 1995.

[19] L. C. Evans and R. F. Gariepy, Measure Theory and Fine Properties of Functions, CRC Press, New York, NY, USA, 1999.

[20] M. K. Kwong, M. Pašić, and J. S. W. Wong, "Rectifiable oscillations in second-order linear differential equations," Journal of Differential Equations, vol. 245, no. 8, pp. 2333-2351, 2008.

[21] M. Onitsuka and J. Sugie, "Uniform global asymptotic stability for half-linear differential systems with time-varying coefficients," Proceedings of the Royal Society of Edinburgh A, vol. 141, no. 5, pp. 1083-1101, 2011.

[22] Ju. L. Daleckii and M. G. Kreĭn, Stability of Solutions of Differential Equations in Banach Space, vol. 43 of Translations of Mathematical Monographs, American Mathematical Society, Providence, RI, USA, 1974.

[23] S. G. Kreı̆n, Linear Differential Equations in Banach Space, vol. 29 of Translations of Mathematical Monographs, American Mathematical Society, Providence, RI, USA, 1971.

[24] A. Pazy, Semigroups of Linear Operators and Applications to Partial Differential Equations, vol. 44 of Applied Mathematical Sciences, Springer, New York, NY, USA, 1983.

[25] A. Favini and E. Obrecht, Differential Equations in Banach Spaces, vol. 1223 of Lectures notes in Mahtematics, Springer, New York, NY, USA, 1985.

[26] L. H. Hao and K. Schmitt, "Fixed point theorems of Krasnoselskii type in locally convex spaces and applications to integral equations," Results in Mathematics, vol. 25, no. 3-4, pp. 290-314, 1994.

[27] C. Avramescu, "Some remarks on a fixed point theorem of Krasnoselskii," Electronic Journal of Qualitative Theory of Differential Equations, vol. 2003, no. 5, pp. 1-15, 2003.

[28] L. T. P. Ngoc and N. T. Long, "On a fixed point theorem of Krasnoselskii type and application to integral equations," Fixed Point Theory and Applications, vol. 2006, Article ID 30847, 24 pages, 2006. 
[29] L. T. P. Ngoc and N. T. Long, "Applying a fixed point theorem of Krasnosel'skii type to the existence of asymptotically stable solutions for a Volterra-Hammerstein integral equation," Nonlinear Analysis: Theory, Methods \& Applications, vol. 74, no. 11, pp. 3769-3774, 2011.

[30] S. Lang, Real and Functional Analysis, Springer, New York, NY, USA, 3rd edition, 1993.

[31] S. Roman, Advanced Linear Algebra, Springer, New York, NY, USA, 2008. 


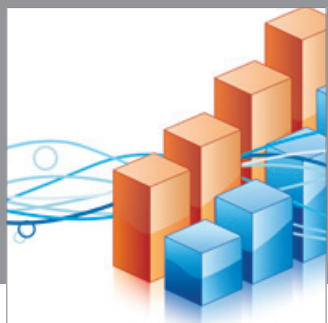

Advances in

Operations Research

mansans

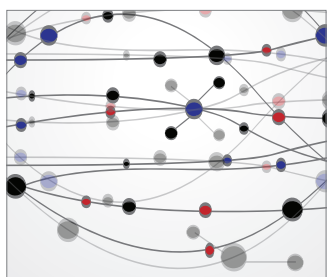

The Scientific World Journal
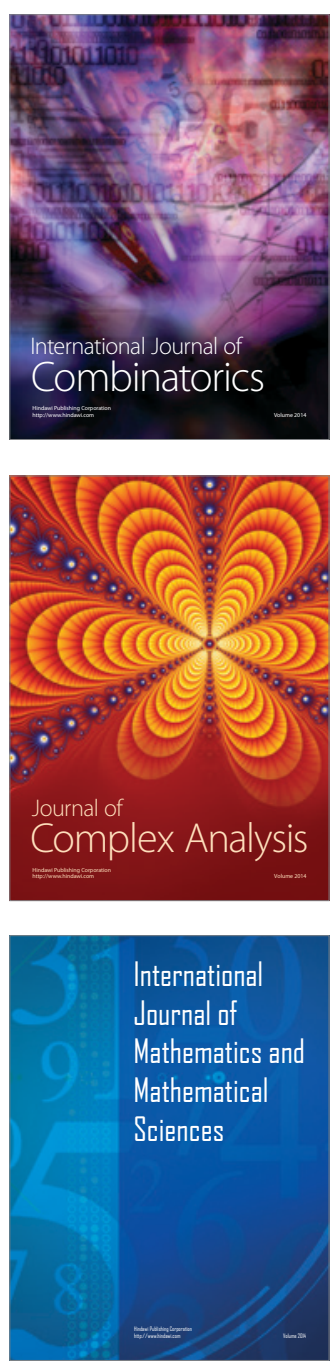
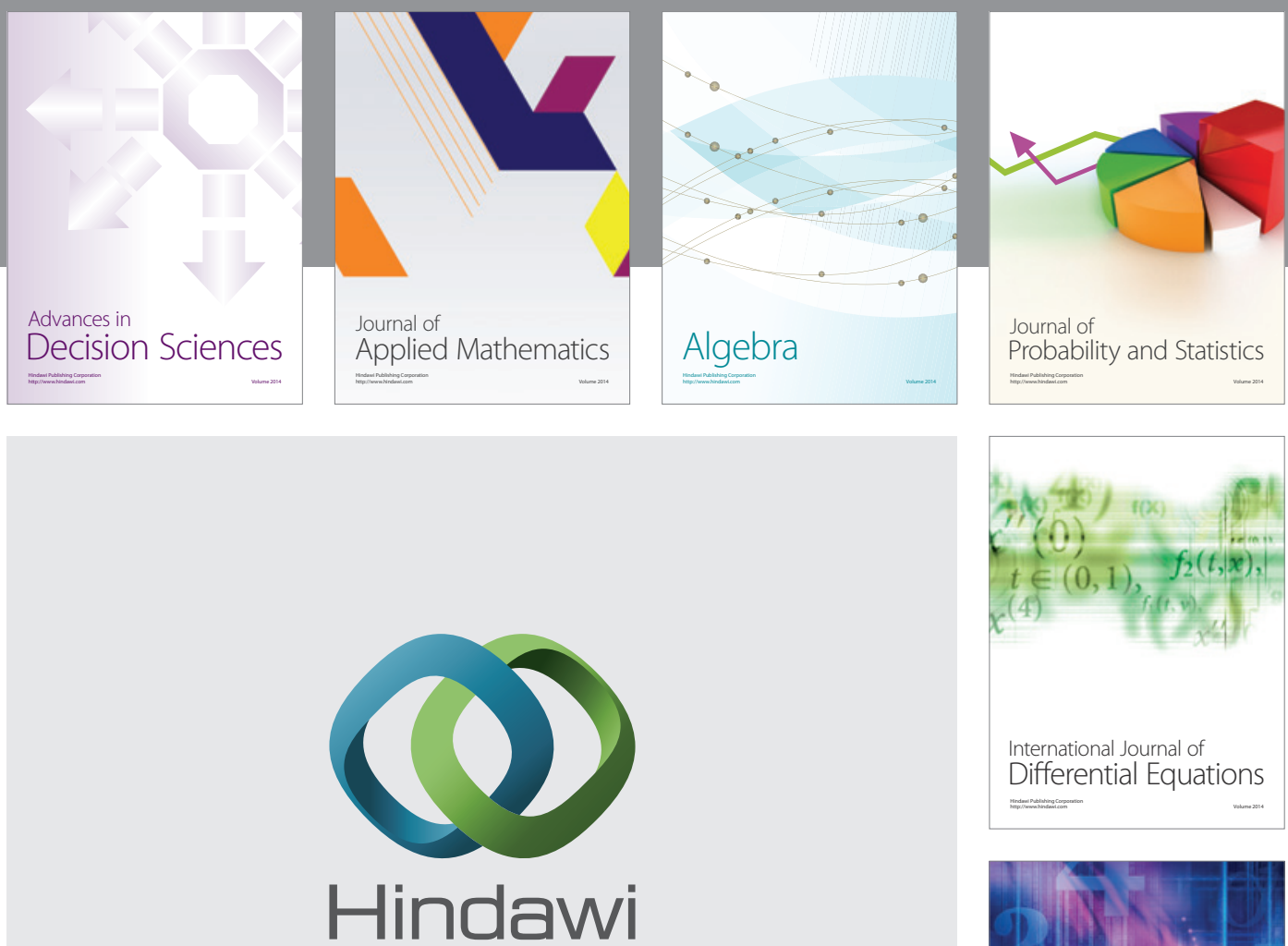

Submit your manuscripts at http://www.hindawi.com
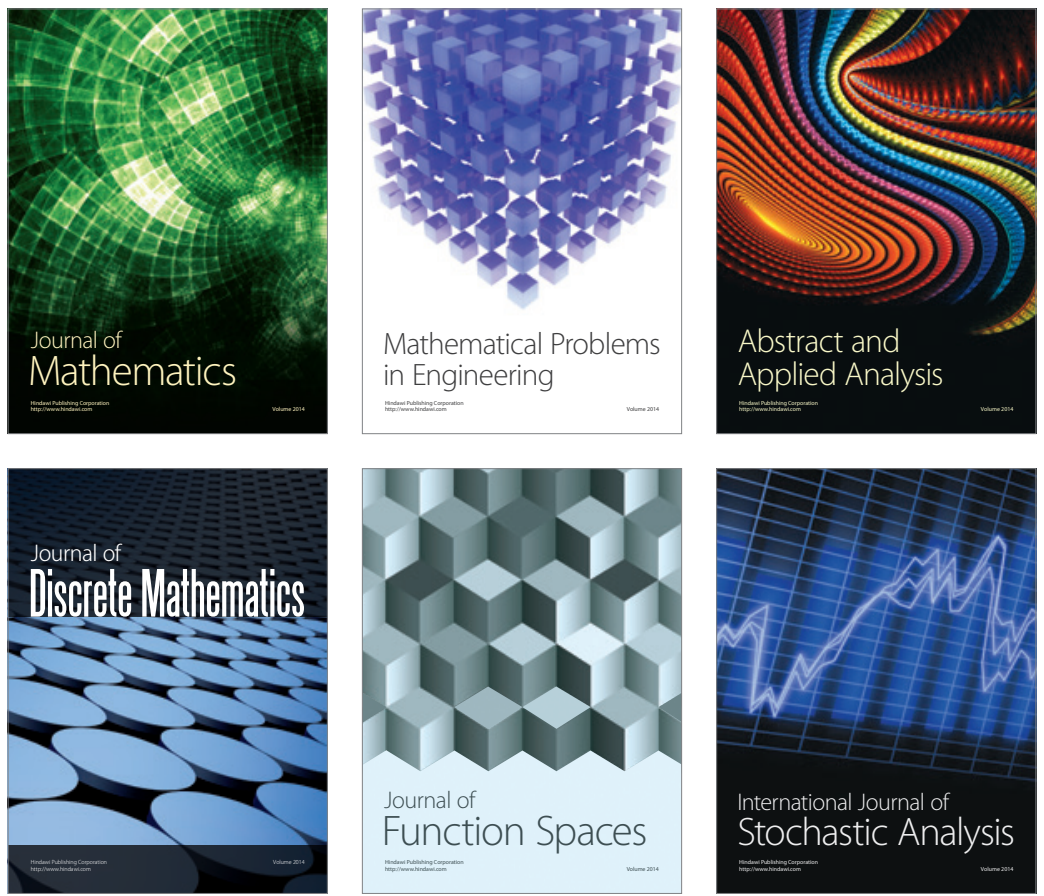

Journal of

Function Spaces

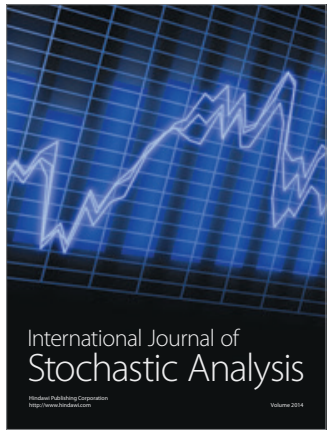

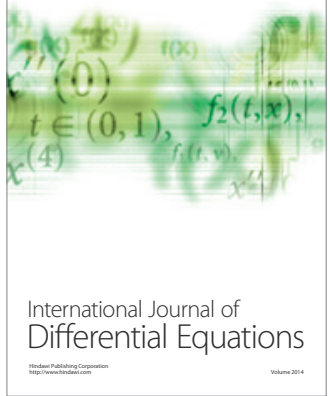
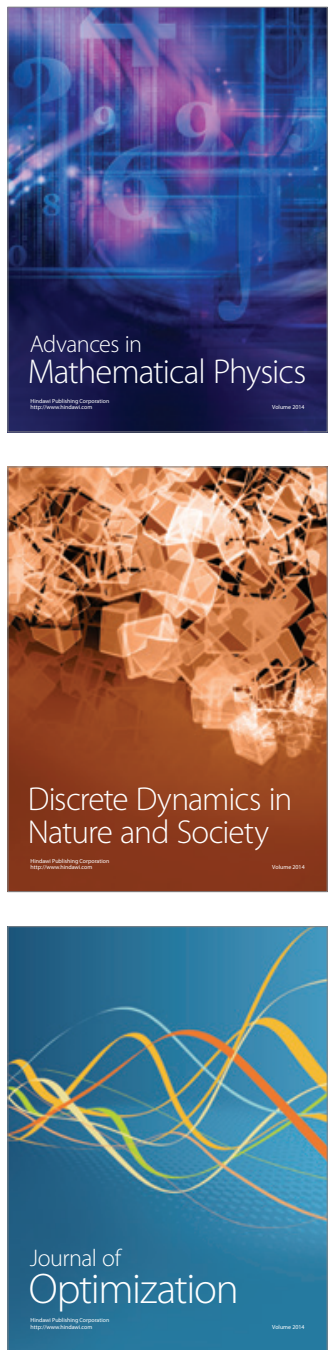